48 (1) | 2019

Varia

\title{
Trazando fronteras: la producción de territorios indígenas en la Amazonía peruana
}

Tracer des frontières : la production de territoires indigènes dans l'Amazonie $p$

éruvienne

Making boundaries: the production of indigenous territories in the Peruvian

Amazon

Danny Pinedo

\section{OpenEdition}

Journals

Edición electrónica

URL: http://journals.openedition.org/bifea/10360

DOI: 10.4000/bifea.10360

ISSN: 2076-5827

\section{Editor}

Institut Français d'Études Andines

\section{Edición impresa}

Fecha de publicación: 1 abril 2019

Paginación: 21-37

ISSN: 0303-7495

\section{Referencia electrónica}

Danny Pinedo, «Trazando fronteras: la producción de territorios indígenas en la Amazonía peruana », Bulletin de l'Institut français d'études andines [En línea], 48 (1) | 2019, Publicado el 08 abril 2019, consultado el 04 noviembre 2020. URL : http://journals.openedition.org/bifea/10360 ; DOI : https:// doi.org/10.4000/bifea.10360

\section{(c) $(1) \odot$}

Les contenus du Bulletin de l'Institut français d'études andines sont mis à disposition selon les termes de la licence Creative Commons Attribution - Pas d'Utilisation Commerciale - Pas de Modification 4.0 International. 


\title{
Trazando fronteras: la producción de territorios indígenas en la Amazonía peruana
}

\author{
Danny Pinedo*
}

\begin{abstract}
Resumen
Este artículo analiza el rol del Estado en la producción de territorios indígenas entre los arakbut de la Amazonía suroriental peruana. La demarcación de las tierras de comunidades nativas y de reservas comunales ha permitido al Estado controlar los espacios y a las poblaciones indígenas de la Amazonía. Los territorios indígenas no solo han facilitado la administración de recursos naturales en función de intereses privados, sino que han generado nuevas nociones de territorio e identidad que exacerban tensiones entre comunidades arakbut. Sin embargo, los arakbut no han sido meras víctimas de las políticas estatales de territorialización de la Amazonía. Ellos han disputado la autoridad territorial del Estado no rechazando sus territorios sino apropiándose de ellos para legalizar sus demandas. Al hacerlo, sin embargo, los arakbut han jugado un papel central en los procesos de formación del Estado en la Amazonía.
\end{abstract}

Palabras clave: arakbut, Estado, identidad, territorio, Amazonía peruana

\section{Tracer des frontières : la production de territoires indigènes dans I'Amazonie péruvienne}

\section{Résumé}

Cet article analyse le rôle de l'État dans la production de territoires indigènes parmi les Arakbut du sud-est de l'Amazonie péruvienne. La démarcation des terres des communautés natives et des réserves communales a permis à l'État de contrôler les espaces et les populations indigènes de l'Amazonie. Les territoires indigènes ont non seulement facilité la gestion des ressources naturelles en fonction d'intérêts privés, mais aussi généré de nouvelles notions de territoire et d'identité qui exacerbent les tensions entre les communautés Arakbut. Cependant, les Arakbut n'ont pas été de simples victimes

* Profesor del Departamento Académico de Antropología de la Universidad Nacional Mayor de San Marcos, Calle Germán Amézaga 375 - Pabellón José Carlos Mariátegui, Ciudad Universitaria, Lima 1. E-mail: dpinedog@unmsm.edu.pe 
des politiques étatiques de territorialisation de I'Amazonie. Ils ont contesté I'autorité territoriale de l'État non pas en rejetant ses territoires mais en se les appropriant pour légaliser leurs revendications. Ce faisant, cependant, les Arakbut ont joué un rôle central dans les processus de formation de l'État en Amazonie.

Mots-clés : Arakbut, État, identité, territoire, Amazonie péruvienne

\title{
Making boundaries: the production of indigenous territories in the Peruvian Amazon
}

\begin{abstract}
This article analyzes the role of the state in the production of indigenous territories among the Arakbut of the southeastern Peruvian Amazon. The demarcation of the lands of native communities and communal reserves has allowed the state to subject Amazonian spaces and indigenous populations to its control. Not only have indigenous territories facilitated the administration of natural resources based on private interests but they have also generated new notions of territory and identity that exacerbated tensions among Arakbut communities. Nevertheless, the Arakbut have not been mere victims of state policies of the territorialization of the Amazon. They have contested the territorial authority of the state not by rejecting its territories but by appropriating them to legalize their territorial claims. In doing so, the Arakbut have played a central role in processes of state formation in the Amazon.
\end{abstract}

Keywords: Arakbut, identity, Peruvian Amazonia, state, territory

\section{INTRODUCCIÓN}

A pesar de su imagen de región de frontera, la Amazonía no ha permanecido al margen del Estado. La Amazonía ha sido sometida al poder del Estado a través de procesos como la misionización, la extracción de recursos naturales y la colonización, en algunos lugares desde épocas tan tempranas como el siglo XVII. Estos procesos se han basado en gran parte en la territorialización del espacio que ha permitido al Estado controlar a las poblaciones y los recursos naturales. En las últimas décadas, esta territorialización ha incluido la producción de territorios indígenas, principalmente mediante la titulación de las tierras de comunidades nativas y la creación de reservas comunales. En este artículo, analizo el papel que las comunidades nativas y la Reserva Comunal Amarakaeri han jugado en la consolidación del poder estatal entre los arakbut de la Amazonía suroriental peruana. El artículo examina también cómo los actores sociales envueltos en estos procesos de territorialización configuraron y reconfiguraron significados que dieron sentido a sus luchas por los recursos naturales y las legitimaron.

1 Esta discusión se basa en el trabajo de campo etnográfico que realicé en cinco comunidades arakbut (Puerto Luz, San José de Karene, Barranco Chico, Boca del Inambari y Shintuya) entre 2011 y 2013. 
Sostengo que la producción de territorios indígenas ha sido esencial para poner el espacio amazónico y a sus pobladores indígenas bajo la autoridad del Estado. Entre los arakbut, la demarcación de las tierras de comunidades nativas y de la Reserva Comunal Amarakaeri, así como su superposición con otras formas territoriales, han permitido al Estado distribuir derechos de propiedad sobre la tierra y los recursos naturales a fin de administrarlos principalmente de acuerdo con los intereses del capital privado. A la vez, la titulación de tierras comunales produjo cambios en las nociones indígenas de territorio e identidad, los cuales tuvieron importantes repercusiones para la movilización política de los arakbut. Sin embargo, los arakbut no han sido meras víctimas de las políticas estatales de territorialización de la Amazonía. Ellos han perseguido sus fines, principalmente la defensa de sus territorios, dentro de los límites de las políticas estatales. Para ello, los arakbut han demandado activamente al Estado la titulación de sus tierras comunales y la creación de la Reserva Comunal Amarakaeri, apelando a diferentes narrativas para legitimar sus demandas. Al hacerlo, sin embargo, han jugado un papel central en los procesos de formación del Estado peruano en la Amazonía².

\section{ESTADO, TERRITORIO E IDENTIDAD}

En este artículo, uso una perspectiva teórica que entiende la territorialidad como la base sobre la cual los Estados demandan autoridad sobre poblaciones y recursos. El geógrafo norteamericano Robert Sack define la territorialidad como «una estrategia espacial para afectar, influenciar o controlar recursos y personas, a través del control de áreas... [E]stá relacionada con la manera en que la gente usa la tierra, se organiza en el espacio y le da significado al lugar» (Sack, 1986: 1-2, mi traducción). Este control estatal tiene lugar a través de la división del territorio en zonas complejas y superpuestas, del reordenamiento de la población y de los recursos de estas zonas, y de la creación de reglas que definen la manera de usar dichas zonas y quiénes pueden hacerlo (Vandergeest \& Peluso, 1995: 387).

Desde esta perspectiva, los territorios indígenas pueden entenderse como tecnologías políticas para expandir la soberanía del Estado sobre áreas ocupadas por poblaciones originarias. Estas tecnologías incluyen prácticas como la demarcación y la titulación de tierras, el mapeo participativo y la planificación del desarrollo (Bryan, 2011; Sletto, 2009). La aplicación de estas tecnologías políticas define no solo los territorios, sino también los derechos asociados a ellos. Conceptualmente, a través del reconocimiento de derechos, el territorio cumple la función de hacer gobernables los espacios. Por ejemplo, el reconocimiento

2 En este artículo, entiendo la presencia del Estado en la Amazonía como el resultado de un proceso de formación del Estado antes que como uno de expansión, desde su centro hacia sus márgenes, de un Estado ya conformado. El concepto de «formación del Estado» implica que el Estado es resultado de los encuentros cotidianos entre individuos o grupos y representantes del Estado (Trouillot, 2001:125). Más que a través de la extensión de instituciones del Estado hacia zonas de frontera, el Estado se construye de manera cotidiana a través de situaciones locales donde los actores sociales luchan por poder, recursos y significados (Krohn-Hansen \& Nustad, 2005:12). 
legal de la propiedad colectiva a los pueblos indígenas determina cuáles derechos pueden ser reivindicados y cuáles no (Bryan, 2011; Hale, 2011). En un contexto neoliberal, este reconocimiento de derechos puede ser funcional al mantenimiento del orden socioespacial necesario para el funcionamiento de los mercados (Hale, 2005; Offen, 2003b).

En esta misma línea, los estudios poscoloniales han puesto especial énfasis en la producción de territorios como un mecanismo para la dominación colonial. Estos estudios proponen que los esfuerzos de los Estados-nación por definir territorios implican la espacialización de las diferencias sociales y étnicas (Mbembe, 2000; Wainwright \& Bryan, 2009). El territorio es parte instrumental de procesos a través de los cuales jerarquías étnico-raciales naturalizan divisiones coloniales (Mbembe, 2000; Mollett, 2011; Quijano, 2000). De este modo, los estudios poscoloniales han cuestionado la idea de que las demandas indígenas por territorio alteran sustancialmente el orden socioespacial de los Estados-nación. Al apropiarse de este orden, los indígenas contribuyen a recrearlo en lugar de desafiarlo (Bryan, 2012; Hale, 2005).

Los territorios no solo son producidos socialmente; también están imbuidos de símbolos y significados que actúan sobre los actores sociales para configurar identidades colectivas. La producción del espacio es una continua práctica discursiva de inclusión y exclusión a través de la cual se construyen diferencias sociales que son la base de identidades colectivas (Bryan, 2012; Herb, 1999; Nagar, 1997). Las toponimias, los relatos históricos y los mapas esquemáticos son estrategias narrativas que transmiten identidad al grupo y sus espacios (Offen, 2003a). Estas estrategias discursivas son usadas a menudo para demostrar las bases históricas y legales de la posesión de la tierra, otorgando autoridad moral a los reclamos por derechos territoriales y movilizando apoyo popular hacia ellos. Desde esta perspectiva, los «territorios indígenas» son espacios que tanto el Estado como los indígenas representan a través de discursos públicos y prácticas políticas (Offen, 2003a).

Estos enfoques resaltan el hecho de que los territorios indígenas son producto de relaciones sociales. En este artículo, busco demostrar cómo las comunidades nativas y las reservas comunales son el resultado de luchas entre representantes del Estado, corporaciones, colonos e indígenas por el control de espacios y recursos en la Amazonía. Desde esta perspectiva, más que solo instrumentos del poder estatal, los territorios indígenas expresan también los intereses y estrategias políticas de los indígenas. Esto hace que los territorios indígenas sean cambiantes y, por lo tanto, tengan un carácter inacabado, desafiando todo intento de definirlos como entidades espaciales estáticas y fijas. Por otro lado, la literatura también enfatiza cómo los territorios indígenas expresan simbólicamente diferencias sociales. En este trabajo demuestro cómo, en su afán por legitimar sus derechos territoriales, diversos actores sociales atribuyen a comunidades nativas y reservas comunales diferentes significados. Estas prácticas discursivas terminan reproduciendo jerarquías étnicas en las que se funda el poder estatal. 


\section{ESTADO Y TERRITORIOS INDÍGENAS EN LA AMAZONÍA}

Durante la Colonia y a lo largo de gran parte de la República, los pobladores indígenas de la Amazonía peruana no fueron considerados sujetos de derecho. Por lo tanto, no se les reconocía ningún derecho, incluyendo derechos de propiedad sobre los territorios que ocupaban. La promulgación en 1957 del Decreto Supremo 03, que adjudicaba tierras a los indígenas a través de la creación de reservas indígenas, significó un gran avance. Sin embargo, esta ley no reconocía la propiedad de la tierra, que se mantenía en manos del Estado. Desde la década de 1960, algunos pueblos indígenas exigieron al Estado la propiedad legal de sus tierras, sobre todo en áreas que soportaban fuertes procesos de colonización3. En respuesta a estas demandas, el reformista gobierno militar del General Juan Velasco Alvarado promulgó en 1974 la Ley de Comunidades Nativas y de Promoción Agropecuaria de Regiones de Selva y Ceja de Selva (Decreto Ley 20653), que reemplazó el Decreto Supremo 03. Por primera vez en la historia del Perú, una ley reconocía a los asentamientos indígenas de la Amazonía como personas jurídicas y les confería derechos de propiedad sobre sus tierras, derechos que tenían el carácter de inalienables, imprescriptibles e inembargables.

Sin embargo, como su nombre lo indica, la ley no solo buscaba otorgar protección legal a las tierras indígenas, sino también impulsar el desarrollo agrícola en la Amazonía. La ley fue promulgada en el contexto de la reforma agraria de Velasco que promovía la modernización del sector agrícola a través de la liquidación de la gran propiedad y su reemplazo por cooperativas (Mayer, 2009). La reforma agraria, sin embargo, dejó fuera a la selva baja, donde la mayoría de grandes latifundios no había sobrevivido al ocaso del auge del caucho. La selva baja quedó sujeta a la Ley de Comunidades Nativas, que fomentaba el desarrollo agrícola a través de la adjudicación de tierras (a comunidades nativas, cooperativas e individuos) y de la producción agropecuaria orientada al mercado.

La Ley de Comunidades Nativas trajo consigo tecnologías políticas del Estado moderno, como la demarcación y el mapeo de tierras comunales, así como la construcción de identidades. La ley incorporó al uso oficial el término «nativo», de claro contenido étnico, para referirse a la población indígena amazónica, que era conocida mediante términos despectivos como «chuncho» y salvaje. La reforma agraria de Velasco había sustituido los términos indio e indígena, que eran de origen colonial y tenían una carga peyorativa, por campesino, un término vinculado al discurso de clase dominante en la época. Sin embargo, este giro retórico no se aplicó a la región amazónica. Nativo, en el sentido de miembro de una comunidad con un territorio comunal demarcado, fue construido como una categoría territorial. La ley fijó la categoría nativo en el espacio, dando lugar a un proceso de indigenización del territorio. De esta manera, a diferencia de las tierras de las comunidades campesinas, las de las comunidades nativas pasaron a ser conocidas como «territorios indígenas». La demarcación territorial y una 
membresía fija hicieron que los complejos territorios e identidades étnicas de los tiempos anteriores al contacto con la sociedad nacional fueran inteligibles a la ley y a la administración regular.

La ley confirió a los indígenas amazónicos derechos de propiedad colectiva sobre las tierras adyacentes a sus principales asentamientos, y no sobre sus territorios étnicos. Los asentamientos indígenas que la ley reconoció como «comunidades nativas» se habían formado mucho antes de la dación de la ley, a través de varios procesos de concentración de población indígena, como misiones, escuelas, fundos, reservas, etc. (Chirif \& García Hierro, 2007). Estos asentamientos indígenas exigieron al Estado títulos colectivos sobre sus tierras. Asimismo, el antropólogo Stefano Varese, a quien el régimen de Velasco había encargado la tarea de elaborar la ley, definió a la comunidad como la unidad social indígena amazónica (Varese, 2018: 430). Barclay \& Santos Granero (1980: 44) sugieren que no titular territorios étnicos fue parte de una estrategia del Estado para promover la fragmentación de los territorios indígenas y la erosión de las identidades étnicas. Así, explican estos autores, la creación de las comunidades nativas «legalizaba» el despojo territorial de los indígenas, ya que al titular solo fragmentos de sus territorios tradicionales convertía automáticamente las tierras que no eran tituladas en tierras del Estado, las que luego podían ser asignadas a la colonización o a concesiones para la explotación de madera, minerales e hidrocarburos. Chirif \& García Hierro (2007: 158), por el contrario, sostienen que el desmembramiento de los territorios e identidades indígenas empezó mucho antes de la promulgación de la Ley de Comunidades Nativas, y que si esta no buscaba titular territorios étnicos fue porque las demandas indígenas por seguridad jurídica provenían mayormente de áreas que, como la Selva Central, habían sido más afectadas por procesos de colonización que fragmentaron los territorios étnicos en áreas muy pequeñas, aisladas y donde la población indígena ya vivía en asentamientos nucleados y rodeados de colonos. Considero que ambas posiciones no son excluyentes. Si bien la ley se adecuó a la realidad de las áreas más colonizadas, donde los territorios indígenas ya estaban fragmentados, terminó atomizando aún más los territorios y etnicidades indígenas.

A la Ley de Comunidades Nativas le siguieron otras que buscaban también consolidar el control del Estado en la Amazonía. En 1975, poco antes del final de su gobierno, Velasco promulgó la Ley Forestal y de Fauna Silvestre (Decreto Ley 21147) que establecía que las tierras con aptitud forestal eran propiedad del Estado y, por lo tanto, no podían titularse en favor de las comunidades nativas. Esta tendencia a recortar los derechos territoriales de los indígenas se acentuó cuando en 1975 el general Francisco Morales Bermúdez derrocó a Velasco mediante un golpe de Estado e inició un proceso de desmantelamiento de sus reformas. El nuevo gobierno militar priorizó la inversión privada en los sectores agropecuario y forestal en la Amazonía, con lo cual el número de comunidades nativas tituladas por año disminuyó progresivamente (Chirif \& García Hierro, 2007: 173). Además, a fin de adecuar la legislación indígena a la Ley Forestal y de Fauna Silvestre, en 1978 Morales Bermúdez derogó la Ley de Comunidades Nativas de 1974 y 
la reemplazó por la Ley de Comunidades Nativas y de Desarrollo Agrario de la Selva y Ceja de Selva (Decreto Ley 22175), que ya no incluía los bosques entre las tierras que podían titularse a nombre de las comunidades, cambiando la propiedad comunal de estas tierras por la figura de la cesión en uso. Sin embargo, Moore (1980: 460) sostiene que este cambio en la ley tuvo también como propósito beneficiar a inversionistas privados interesados en la minería aurífera y la agroindustria.

En compensación por la exclusión de los bosques de las tierras tituladas a favor de las comunidades nativas, la Ley Forestal y de Fauna Silvestre creó las Reservas Comunales (Smith \& Pinedo, 2003: 19). En 1990, las reservas comunales fueron incluidas en el Sistema Nacional de Áreas Naturales Protegidas por el Estado (SINANPE) y en 1997 la Ley de Áreas Naturales Protegidas (Ley 26834) las categorizó como áreas naturales protegidas de uso directo, es decir, que permiten el aprovechamiento de recursos naturales. Sin embargo, esta última las distinguió de otras áreas de uso directo al señalar que su principal objetivo era promover la conservación de la fauna y de la flora para el beneficio de las comunidades adyacentes. Además, debían ser coadministradas por el Estado y las comunidades beneficiarias, aunque la propiedad permanecía en poder del Estado. Los indígenas vieron las reservas comunales como una oportunidad de recuperar el control sobre áreas que consideraban parte de sus territorios ancestrales pero que no llegaron a ser tituladas a nombre de comunidades nativas. Dicho control les permitiría proteger sus territorios de la invasión de madereros ilegales, compañías mineras y colonos (Newing \& Wahl, 2004). Sin embargo, los conservacionistas de la agencia estatal de áreas protegidas creían que, por tratarse de espacios de gran biodiversidad, las áreas propuestas para reservas comunales debían ser protegidas a través de categorías de protección más estricta como los parques nacionales. Además, no veían con buenos ojos que las comunidades locales coadministraran las reservas comunales, pues esto les restaba control sobre dichas áreas.

$\mathrm{Ni}$ los títulos comunales ni las reservas comunales reconocen a los indígenas la propiedad sobre los recursos naturales (bosques, minerales, hidrocarburos, cuerpos de agua, fauna y flora, etc.), que son definidos como propiedad del Estado. Esto dio origen a un proceso de superposición de territorialidades que ha desempoderado aún más a las comunidades nativas. Por ejemplo, la Ley General de Minería (Decreto Supremo 014-92-EM) permitió establecer concesiones de minerales metálicos sobre áreas agrícolas intangibles, lo cual afectó tanto a comunidades nativas como a áreas naturales protegidas. La legislación también permitió otorgar contratos de operaciones de hidrocarburos sobre áreas tituladas a comunidades nativas y sobre áreas naturales protegidas de uso directo, aunque esto último depende de la compatibilidad entre las actividades extractivas y la categoría, la zonificación y el plan maestro del área protegida. A continuación, el artículo analiza el impacto que estos procesos de territorialización tuvieron entre los arakbut. 


\section{LAS COMUNIDADES ARAKBUT}

Las actuales comunidades arakbut se formaron entre fines de la década de 1960 e inicios de la de 1970, con los remanentes de varias malocas que escaparon de la misión de Shintuya, donde habían sido concentradas por misioneros dominicos a finales de la década de 1950. Sin embargo, poco tiempo después de su formación, estos asentamientos fueron invadidos por empresas petroleras, compañías mineras y miles de colonos mineros. Esto suscitó la movilización colectiva de los arakbut, no solo para expulsar físicamente a los invasores, sino también para exigir al Estado la titulación de sus tierras. Después de varios años de demandas, el Estado reconoció los asentamientos arakbut como comunidades nativas y les concedió títulos colectivos de las tierras que ocupaban. Salvo Shintuya, que fue reconocida en 1974 y titulada en 1978, la mayoría de las comunidades arakbut fue reconocida y titulada entre 1980 y 1988. Si bien la titulación de tierras permitió a los arakbut defender sus territorios, también produjo cambios en sus nociones de territorio y de identidad que terminaron acentuando tensiones entre comunidades. Asimismo, otras formas territoriales del Estado se superpusieron a los territorios comunales, lo cual recortó los derechos territoriales de los arakbut.

Los arakbut de Puerto Luz, por ejemplo, lograron en 1986 que el Estado demarcara en su favor 56873 ha, de las cuales solo fueron tituladas 38784 ha de tierras cultivables, mientras que el resto (18 089 ha de tierras forestales) les fue otorgado mediante contrato de cesión en uso. Una de las principales consecuencias de la demarcación y de la titulación de las tierras de los asentamientos arakbut fue la transformación de la concepción arakbut de territorio, de uno con fronteras fluidas y cambiantes a uno espacialmente fijo ${ }^{4}$. A su vez, la comunidad nativa cambió la forma en que los arakbut ganaban el acceso al territorio comunal y a los recursos que se encontraban dentro de él, pues dicho acceso quedó legalmente restringido a aquellos individuos reconocidos como miembros de una comunidad. El área demarcada o titulada de una comunidad nativa en particular se convirtió en el territorio discreto de sus miembros, del cual no solo los no arakbut sino también los arakbut de otras comunidades quedaban excluidos. A partir de entonces empezaron a haber tensiones por recursos naturales entre comunidades colindantes. Por ejemplo, en una oportunidad, debido al temor de que no tuvieran suficiente caña brava para la elaboración de flechas, los arakbut de Puerto Luz negaron a los de la vecina San José de Karene el acceso a cañaverales localizados al interior de sus límites comunales (Gray, 1997).

Tanto la movilización colectiva como la demarcación territorial fomentaron entre los arakbut un sentido de pertenencia a la localidad que fue la base de la formación de identidades comunitarias. Los indígenas de habla harakbut ya no solo se identificaban como hablantes de uno de los siete dialectos de esta

4 Antes del contacto, miembros de malocas y de pueblos indígenas vecinos que eran considerados aliados podían penetrar en el territorio discreto de una maloca, mientras que aquellos considerados enemigos no eran bienvenidos (Gray, 1997: 99). Sin embargo, las alianzas eran inestables y un grupo podía pasar de aliado a enemigo y viceversa. Esto coincide con descripciones de visiones indígenas del territorio en términos relacionales (Echeverri, 2004). 
lengua (p. ej., arakbut, wachiperi o arasaeri), o como miembros de un clan o grupo residencial, sino también, y a veces principalmente, como gente de tal o cual comunidad. Esta identidad comunitaria permitió a los arakbut forjar lazos de solidaridad que facilitaron su movilización colectiva contra nuevas invasiones de mineros y corporaciones. Offen (2003a) señala que prácticas como el mapeo de territorios comunales, a través de ejercicios participativos de memoria de la historia de las comunidades, crean identidad. Sostengo que, en el caso de las comunidades arakbut, la identidad comunitaria fue construida a través tanto de la movilización de los arakbut para defender sus territorios comunales como de la introducción de fronteras territoriales claras y fijas.

Esta misma identidad comunal, sin embargo, agudizó tensiones entre comunidades que tenían su origen en antiguas rivalidades entre malocas. Estas tensiones constituyeron un obstáculo para la formación de redes intercomunitarias de defensa territorial cuando el número de mineros invasores se incrementó significativamente. La participación de activistas de los derechos indígenas, entre ellos varios antropólogos, fue crucial para limar estas asperezas y crear coaliciones que eventualmente terminaron en la creación en 1982 de la Federación Nativa del Río Madre de Dios y Afluentes (FENAMAD) (Pinedo, 2017). Así, a pesar de que la Ley de Comunidades Nativas no se proponía deliberadamente erosionar identidades étnicas, pues éstas ya se encontraban fragmentadas, terminó acentuando la atomización entre los indígenas.

Pese a que la titulación de tierras comunales trajo consigo una visión modernista del territorio caracterizada por límites fijos, los arakbut no abandonaron del todo sus propias nociones. En primer lugar, a pesar de que el estatus de miembro de una comunidad nativa quedó legalmente sujeto a la residencia permanente en ésta, los arakbut han continuado moviéndose de una comunidad a otra. Esta movilidad se debe al establecimiento de alianzas matrimoniales, a la búsqueda de trabajo temporal en la extracción de oro o a la necesidad de resolver conflictos. En segundo lugar, los arakbut pueden considerar que una zona es parte de su territorio incluso si no ha sido incluida en los límites titulados de su comunidad. Por lo tanto, pueden extraer recursos de ella y defenderla de amenazas externas. Esto sucede con la zona conocida como La Cumbre, que los arakbut de Puerto Luz consideran parte de su territorio a pesar de que se encuentra en la zona de amortiguamiento de la Reserva Comunal Amarakaeri y no dentro del territorio titulado de la comunidad. Los arakbut de Puerto Luz han usado La Cumbre como zona de caza y recolección desde antes que pasara a formar parte de la reserva comunal.

La titulación de tierras comunales no fue un impedimento para que el Estado otorgara a terceros denuncios mineros sobre comunidades arakbut. La nueva legislación minera, promulgada entre fines de la década de 1970 y principios de la de 19805, hizo posible que medianos y grandes mineros solicitaran denuncios que en su mayoría se superpusieron a territorios de comunidades arakbut. A

5 Ley de Promoción Aurífera (Decreto Legislativo 22178), promulgada en 1978 durante la administración de Morales Bermúdez, y Ley General de Minería (Decreto Legislativo 109), dada en 1981 por el segundo gobierno de Fernando Belaúnde Terry. 
su vez, miles de mineros informales y, por lo tanto sin derecho legal, alquilaron los denuncios de los mineros formales e invadieron tierras de comunidades, dando lugar a conflictos que desembocaron en agresiones físicas y asesinatos de indígenas arakbut (Pacuri \& Moore, 1992: 24). Para hacer frente a este problema, los arakbut pusieron en práctica dos estrategias. Primero, en 1986 la FENAMAD presentó al Congreso de la República una propuesta de ley que buscaba garantizar a los indígenas el aprovechamiento exclusivo de los recursos auríferos dentro de sus territorios comunales. Pese a que la propuesta fue convertida en proyecto de ley, los parlamentarios priorizaron los intereses de grupos de poder vinculados a la minería y el proyecto terminó siendo archivado (Pacuri \& Moore, 1992: 20). Segundo, los arakbut buscaron formular sus propios denuncios mineros dentro de sus linderos comunales y así evitar que sus tierras fueran denunciadas por mineros foráneos. Sin embargo, las comunidades perdieron la mayoría de estos denuncios debido a que les fue muy difícil cumplir con las exigencias que la ley establecía para mantener su vigencia (Pacuri \& Moore, 1992: 38).

La presencia de colonos mineros en las tierras de los arakbut desencadenó entre estos últimos respuestas que implicaron tanto rupturas como continuidades. Cuando los arakbut de Puerto Luz descubrieron depósitos aluviales de oro en La Cumbre, los explotaron en pequeña escala y esporádicamente. Esto fue aprovechado por mineros foráneos que invadieron la zona a principios de la década de 2000. Los arakbut buscaron entonces desalojar a los invasores, lo que llevó a varios enfrentamientos violentos. El conflicto terminó cuando los mineros ofrecieron pagar una renta, o «regalía» como la llaman localmente, a fin de continuar explotando los yacimientos de La Cumbre. Los arakbut empezaron entonces a alquilar La Cumbre y otras zonas ubicadas dentro de las tierras tituladas de la comunidad a mineros foráneos, a quienes llaman «invitados». La relación entre los arakbut y los colonos mineros, que penetraron por primera vez al territorio arakbut en la década de 1960, no siempre ha estado marcada por el conflicto6. Los arakbut no solo aprendieron de los mineros cómo trabajar el oro aluvial, sino que recibieron de ellos herramientas a cambio del permiso de extraer oro en sus territorios. Desde entonces, los arakbut han utilizado el alquiler de tierras a los invitados como un mecanismo para hacer frente a una presencia cada vez mayor y permanente de colonos. Arakbut y colonos mineros incluso establecen vínculos de amistad y compadrazgo para fortalecer su relación (Pinedo, 2014). Por lo tanto, las tierras comunales de los arakbut no siempre están cerradas a los foráneos.

De esta manera, lo que hoy podríamos considerar «territorio arakbut» es un híbrido de diferentes formas de territorialidad que se superponen, se combinan y tienen efectos ambiguos. La organización comunal introdujo una noción de territorialidad con límites definidos y fijos, pero que coexiste con concepciones fluidas del territorio. También produjo, sin proponérselo, una identidad comunitaria

6 La relación entre los arakbut y la población andina se remonta al período incaico, cuando los arakbut y otros pueblos indígenas de la Amazonía suroriental establecieron alianzas políticas y redes de comercio con los incas (Lyon, 1984). 
que, por un lado, ha facilitado la acción colectiva contra las amenazas externas y, por otro, ha generado fricciones entre comunidades vecinas sobre el control de recursos naturales. Los arakbut defienden sus territorios de foráneos, pero también aceptan la presencia de estos y sus demandas de recursos como un mecanismo de resolución de conflictos o de acceso a capital. De otro lado, las comunidades nativas arakbut son un ejemplo de cómo la legislación de comunidades nativas, al crear fronteras administrativas y fijar conjuntos de poblaciones indígenas a ellas, busca racionalizar el gobierno del espacio y de la población en la Amazonía. Las fronteras difusas de los asentamientos arakbut eran particularmente problemáticas para la administración estatal, puesto que impedían establecer derechos de propiedad. Pero si bien los títulos comunales reconocieron derechos de propiedad a los arakbut, éstos se limitaron a la tierra, dejando los que conciernen a los recursos naturales susceptibles de ser asignados a intereses privados.

\section{LA RESERVA COMUNAL AMARAKAERI}

En 1989, los delegados indígenas que asistían al VI Congreso de la FENAMAD en la comunidad de Shintuya acordaron exigir al Estado la creación de una reserva comunal sobre el área que el pueblo arakbut considera su territorio ancestral. La reserva fue propuesta como una estrategia para impedir que el territorio tradicional de los arakbut, que constituye la principal fuente de recursos para su sustento, se redujera aún más debido a la incursión de mineros y madereros ilegales. Los arakbut pensaban que la creación de una reserva comunal no solo prohibiría toda actividad extractiva a gran escala dentro de estos territorios, los cuales habían quedado fuera de las áreas tituladas a las comunidades nativas arakbut, sino que les devolvería cierto control sobre ellos. Sin embargo, la propuesta de creación de la reserva se topó con una férrea resistencia por parte de funcionarios, quienes más bien buscaban destinar el área ya sea a categorías de conservación más estrictas o a actividades petroleras. Esta resistencia desencadenó tensiones entre comunidades arakbut, la FENAMAD y activistas por los derechos indígenas, de un lado, y el Estado y una corporación petrolera, del otro. Los varios discursos en los que las posturas a favor o en contra de la reserva fueron enmarcadas evidencian los diferentes significados que los grupos de interés involucrados dieron a la reserva.

Para legitimar sus demandas por la creación de la reserva comunal, los arakbut buscaron conformarlas a dos discursos hegemónicos en la época: el discurso étnico y el discurso ambientalista. En primer lugar, los arakbut tuvieron que adecuarse a visiones del indígena amazónico que establecen una relación clara y continua entre etnicidad (cultura y lengua) y territorio. La capacidad de los arakbut para afirmar derechos sobre su territorio tradicional dependía de probar el vínculo histórico entre su existencia como pueblo indígena y el espacio que reclamaban como suyo. La FENAMAD encargó entonces a antropólogos la conducción de estudios que revelaran los elementos culturales que demuestren que el área propuesta para la reserva efectivamente correspondía al territorio de los pueblos de lengua harakbut desde épocas anteriores a la creación del Estado peruano. 
Así, la creación de la reserva fue justificada apelando a su condición de «territorio ancestral» de los arakbut. Un informe técnico que contenía la propuesta para crear la reserva sostenía que:

El área propuesta para la creación de la Reserva Comunal Amarakaeri forma parte del territorio étnico tradicional de dicho grupo étnico. En ese sentido, a través del presente estudio, por primera vez se ha podido reconstruir su estado previo al contacto con la sociedad nacional en los años 40. Empleamos para ello diferentes fuentes históricas y la información oral (FENAMAD, 1992: 143).

Debido a que la ley había definido las reservas comunales como áreas protegidas para el manejo de fauna silvestre como fuente tradicional de alimento, los conservacionistas de la Intendencia de Áreas Naturales Protegidas (IANP), en ese entonces la agencia estatal encargada de la administración de dichas áreas, advirtieron a los indígenas que solo actividades de subsistencia que estuvieran en consonancia con prácticas indígenas tradicionales serían consideradas compatibles con los objetivos de la reserva. En ese contexto, los arakbut enfatizaron la importancia de la reserva comunal para satisfacer sus necesidades de subsistencia. El concepto de «actividades de subsistencia» tuvo sin embargo diferentes significados para los conservacionistas y los indígenas. El marco usado por los conservacionistas para justificar la creación de la reserva no incluía actividades extractivas, a pesar de que los arakbut venían practicando la minería aurífera por décadas. Ellos defendieron la tesis de que la minería aurífera es parte de sus actividades de subsistencia, puesto que los ingresos que se obtienen de ella son usados principalmente para satisfacer necesidades primarias, tales como alimentos manufacturados, ropa, educación, herramientas, etc.

Los arakbut también adoptaron el discurso ambientalista para legitimar sus reclamos por la creación de la reserva comunal. Cuando una autoridad estatal cuestionó la extensión solicitada para la reserva, los arakbut respondieron:

Queremos asegurar la extracción racional de una manera sostenible para conservar las diferentes variedades de flora y fauna necesarias para el mantenimiento de nuestras culturas (FENAMAD, 1996: 17).

Los arakbut argumentaron que la reserva comunal protegería zonas importantes no solo por su contribución al sustento de las comunidades nativas, sino también por sus servicios ecológicos. Los funcionarios de la IANP señalaron que la principal función de la reserva debía ser la protección de importantes zonas de cabecera de cuenca de la región de Madre de Dios. Los indígenas respondieron que este criterio coincidía con su percepción de las cabeceras de cuenca como lugares para la reproducción de la vida que no debían ser perturbados por la presencia humana. Así, los arakbut estuvieron de acuerdo en que las cabeceras de cuenca debían permanecer dentro de una zona de protección estricta.

Luego de 13 años de movilizaciones indígenas, en 2002 el Estado peruano finalmente accedió a crear la Reserva Comunal Amarakaeri. Con una extensión de 402 335,62 ha, la reserva beneficiaría a 8 comunidades circundantes: 
Puerto Luz, San José de Karene, Barranco Chico, Shintuya, Boca Isiriwe, Puerto Azul, Diamante y Shipetiari. Sin embargo, asociar la reserva con economías tradicionales de subsistencia y con protección ambiental no fue suficiente para bloquear otras territorialidades promovidas por intereses más poderosos. Así, en 2005, el mismo Estado que había creado la reserva comunal 3 años antes otorgó a Hunt Oil Company, una empresa petrolera con sede en Texas, Estados Unidos, una concesión para explorar petróleo y gas en el Lote 76, un área de 1434026 ha que se superponía a casi la totalidad del territorio de la reserva comunal y al de la mayoría de sus comunidades beneficiarias. Los dirigentes de la FENAMAD señalaron que las comunidades no fueron consultadas sobre la concesión del Lote 76, a pesar de que el Estado estaba obligado a hacerlo en virtud de haber ratificado en 1993 el Convenio 169 de la Organización Internacional del Trabajo. Esta superposición de derechos dio inicio a varios años de fricciones entre el Estado y la federación.

Los líderes de la FENAMAD tenían la esperanza de que la zona de protección estricta pondría alto a las aspiraciones de Hunt Oil en las zonas ambientalmente más sensibles de la reserva comunal. De acuerdo con la legislación de áreas protegidas, el Estado no puede autorizar actividades de hidrocarburos en las partes de una reserva comunal designadas por el plan maestro como zonas de protección estricta. Sin embargo, el jefe de la IANP cuestionó la primera versión del plan maestro de la reserva, que había sido aprobada en abril del 2007 con participación indígena, y condicionó su aprobación a la eliminación de la zona de protección estricta. Cediendo a presiones provenientes de las más altas esferas del poder, los representantes de la IANP presentaron una nueva propuesta de plan maestro que reemplazaba la zona de protección estricta por una zona silvestre que abarcaba solo las cabeceras de cuenca. Los funcionarios de la IANP hicieron otros cambios en el plan maestro a fin de facilitar las actividades de hidrocarburos en su interior, aunque aseguraron a los indígenas que no se permitiría ningún tipo de intervención humana dentro de la nueva zona silvestre. El plan maestro fue aprobado con las enmiendas en una reunión llevada a cabo el 28 de diciembre de 2007, fecha elegida convenientemente, ya que por tratarse de fin de año no permitió la presencia de cuatro de los ocho representantes indígenas del Comité de Gestión de la reserva y de los técnicos y asesores legales de la FENAMAD, quienes por esos días se encontraban de vacaciones.

La FENAMAD buscó tenazmente impedir el ingreso de Hunt Oil a la reserva comunal. Organizó manifestaciones callejeras de protesta, amenazó a la compañía con expulsarla físicamente de la reserva e interpuso en 2009 una demanda de acción de amparo contra ella. También recurrió al audiovisual como parte de una campaña mediática contra la petrolera. Con tal fin, se asoció con el realizador y ambientalista canadiense David Suzuki para producir The Real Avatar, un episodio de su programa The Nature of Things que salió al aire en 2011 y que narraba cómo la compañía texana dividía a los harakbut con el propósito de allanar el camino para su ingreso a la reserva. El título del episodio hacía clara alusión a Avatar, la taquillera película hollywoodense que cuenta la historia de la invasión humana de Pandora, un satélite rico en minerales y habitado por una tribu indígena llamada 
na'vi. El video fue proyectado varias veces para generar el apoyo de la opinión pública, no solo en Puerto Maldonado sino también en Lima. Ya en 2010, Avance Indígena, revista institucional de la FENAMAD, había acompañado una nota sobre la defensa de la reserva comunal con el fotomontaje de un rostro, el cual mostraba en una de sus mitades a un na'vi y en la otra a Jaime Corisepa, en ese entonces presidente de la federación.

Para obtener la aprobación de Shintuya y Puerto Luz a sus actividades de exploración, Hunt Oil recurrió a dos estrategias. Primero, la empresa presentó como una muestra de su generosidad la compensación por daños y perjuicios que los trabajos de sísmica ocasionarían en los territorios comunales, a pesar de que estaba legalmente obligada a ello. Entre 2011 y 2012, Hunt Oil realizaba a favor de Puerto Luz una dotación mensual de combustible para su generador eléctrico, campañas de salud, evacuación de enfermos y entrega de regalos (juguetes y panetones) en Navidad. Segundo, desconociendo el rol de la FENAMAD como representante de los intereses de las comunidades nativas, Hunt Oil se acercó a Shintuya y a Puerto Luz para negociar la compensación de manera directa. En sus negociaciones con ambas comunidades, la empresa puso especial cuidado en resaltar que la ley garantizaba la autonomía de las comunidades nativas para tomar sus decisiones. Como resultado, aquellos arakbut que se inclinaron a favor del ingreso de la petrolera a sus comunidades se valieron del discurso de la autonomía para justificar su posición. Obviamente, esto originó divisiones al interior de Shintuya y de Puerto Luz, así como tensiones entre estas comunidades y la FENAMAD. Algunos miembros de estas comunidades llegaron a afirmar que si los dirigentes de la FENAMAD se oponían a una negociación directa entre las comunidades y la empresa era para beneficiarse ellos mismos de sus resultados. Estas tensiones debilitaron la campaña de la FENAMAD en contra de la Hunt Oil.

Todos los intentos de frenar el avance de Hunt Oil resultaron infructuosos, pues la compañía finalmente ingresó a la reserva comunal para realizar trabajos de exploración. La empresa abrió 18 líneas sísmicas con 20000 puntos de detonación a lo largo de la parte sur de la reserva, que cruzaban también parcialmente los territorios de las comunidades de Shintuya y de Puerto Luz, lo que suscitó un conflicto adicional de territorialidades, esta vez entre el Lote 76 y las comunidades nativas. A fines de 2014, Hunt Oil abrió un pozo exploratorio en la reserva. Sin embargo, los resultados de la perforación no fueron los esperados y devolvió el Lote 76 en agosto de 2017.

A pesar de que Hunt Oil finalmente se retiró de la reserva comunal, su presencia evidenció lo ambiguas que pueden resultar las reservas comunales. Los arakbut vieron la Reserva Comunal Amarakaeri como una oportunidad para recuperar el control sobre extensiones de su territorio que no fueron incluidas en las tierras tituladas a sus comunidades nativas. Sin embargo, las reservas comunales son al mismo tiempo instrumentos de control estatal del espacio amazónico. Ellas reconocen a las comunidades nativas beneficiarias el derecho a aprovechar los recursos de flora y fauna, pero no derechos de propiedad sobre ellos. Además, como lo evidencia el caso de Amarakaeri, el Estado tiene el poder para cambiar la 
zonificación de las reservas comunales en función de intereses privados. Asimismo, el Estado buscó excluir a los arakbut de sus propios territorios exigiéndoles como requisito para la creación de la reserva amoldarse a imágenes idílicas de lo indígena, como economía de subsistencia, tradición y conservación. Con el fin de legitimar sus demandas, los arakbut adoptaron y recrearon estas narrativas de indigeneidad producidas por el propio Estado. Por otro lado, el mismo discurso de autonomía comunitaria, en un nivel sirvió a los intereses de los arakbut, pero en otro permitió a Hunt Oil debilitar la resistencia indígena a su proyecto petrolero.

\section{CONCLUSIONES}

El caso de las comunidades nativas arakbut y de la Reserva Comunal Amarakaeri me permite concluir que los territorios indígenas en la Amazonía peruana no son únicamente estrategias para someter a esta región y a sus habitantes indígenas al poder del Estado, ni solamente el resultado del reconocimiento estatal de las demandas indígenas. En realidad, expresan ambos aspectos. Mediante la demarcación de fronteras claras y fijas, las comunidades nativas y las reservas comunales volvieron inteligibles para el Estado los difusos y cambiantes territorios e identidades indígenas tradicionales. La definición de fronteras territoriales y étnicas permite al Estado el reconocimiento de ciertos derechos de propiedad, pero también el desconocimiento de otros, facilitando su control de las poblaciones indígenas y su acceso a la tierra y a los recursos naturales. Además, la demarcación y la titulación de las tierras comunales tuvieron el inesperado efecto de alentar entre los arakbut la formación de nociones atomizadas y fijas de territorio e identidad. Esto, a su vez, tuvo impactos ambiguos. Por un lado, afianzó la cohesión comunal frente a las amenazas de colonos y empresas mineras; por otro, avivó viejas rivalidades entre comunidades que fueron aprovechadas por una empresa petrolera.

De otro lado, antes que el Estado, han sido los pueblos indígenas los que han promovido la titulación comunal y la creación de reservas comunales sobre sus territorios ancestrales. Por lo tanto, en lugar de ser simplemente receptores de políticas estatales, los indígenas amazónicos son promotores activos de estas. Ellos han abrazado esfuerzos de construcción del Estado cuando éstos les han resultado ventajosos, aprovechando su carácter fragmentario y sus contradicciones internas. El control estatal de la Amazonía a través de la producción de territorios indígenas evidencia pues que el Estado y los indígenas amazónicos tienen un carácter mutuamente constitutivo. Al demandar derechos legales, los arakbut no solo defienden la integridad de sus territorios, sino que contribuyen a aumentar la soberanía y la legitimidad del moderno Estado territorial en la Amazonía. Así, pues, en la Amazonía, la línea que separa al Estado de los pueblos indígenas es borrosa, cambiante y sujeta a una continua producción. 


\section{Referencias citadas}

BARCLAY, F. \& SANTOS GRANERO, F., 1980 - La conformación de las comunidades amuesha: la legalización de un despojo territorial. Amazonía Peruana, 3 (5): 43-74.

BRYAN, J., 2011 - Walking the Line: Participatory Mapping, Indigenous Rights, and Neoliberalism. Geoforum, 42 (1): 40-50.

BRYAN, J., 2012 - Rethinking Territory: Social Justice and Neoliberalism in Latin America's Territorial Turn. Geography Compass, 6 (4): 215-226.

CHIRIF, A. \& GARCÍA HIERRO, P., 2007 - Marcando territorio: progresos y limitaciones de la titulación de territorios indígenas en la Amazonía, 340 pp.; Copenhague: Grupo Internacional de Trabajo sobre Asuntos Indígenas-IWGIA.

ECHEVERRI, J. A., 2004 - Territorio como cuerpo y territorio como naturaleza: ¿diálogo intercultural? In: Tierra adentro: territorio indígena y percepción del entorno (A. Surrallés \& P. García Hierro, eds.): 259-275; Copenhague: Grupo Internacional de Trabajo sobre Asuntos Indígenas-IWGIA.

FENAMAD, 1992 - Informe técnico sobre la propuesta para el establecimiento de la Reserva Comunal Amarakaeri; Puerto Maldonado: Dirección Subregional Agricultura Madre de Dios y Secretaría Regional de Asuntos Productivos Extractivos Región Inka.

FENAMAD, 1996 - Reserva Comunal Amarakaeri. Avance Indígena, 5 (10): 11, 17-18.

GRAY, A., 1997 - Indigenous Rights and Development: Self-determination in an Amazonian Community, 354 pp.; Nueva York: Berghahn Books.

HALE, C. R., 2005 - Neoliberal Multiculturalism: The Remaking of Cultural Rights and Racial Dominance in Central America. PoLAR: Political and Legal Anthropology Review, 28 (1):10-28.

HALE, C. R., 2011 - Resistencia para que? Territory, Autonomy and Neoliberal Entanglements in the 'Empty Spaces' of Central America. Economy and Society, 40 (2):184-210.

HERB, G. H., 1999 - National Identity and Territory. In: Nested Identities: Nationalism, Territory, and Scale (G. H. Herb \& D. H. Kaplan, eds.): 9-30; Lanham: Rowman and Littlefield.

KROHN-HANSEN, C. \& NUSTAD, K. G., 2005 - Introduction. In: State Formation: Anthropological Perspectives (C. Krohn-Hansen \& K. G. Nustad, eds.): 3-26; London: Pluto Press.

LYON, P. J., 1984 - An Imaginary Frontier; Prehistoric Highland-lowland Interchange in the Southern Peruvian Andes. In: Networks of the Past. Regional Interaction in Archaeology (P. D. Francis, F. J. Kense \& P. G. Duke, eds.): 3-18. Calgary: University of Calgary.

MAYER, E., 2009 - Cuentos feos de la reforma agraria peruana, 354 pp.; Lima: Instituto de Estudios Peruanos.

MBEMBE, A., 2000 - At the Edge of the World: Boundaries, Territoriality, and Sovereignty in Africa. Public Culture, 12 (1): 259-284.

MOLLETT, S., 2011 - Racial Narratives: Miskito and Colono Land Struggles in the Honduran Mosquitia. Cultural Geographies, 18 (1): 43-62.

MOORE, T., 1980 - Transnacionales en Madre de Dios: implicancias para las comunidades nativas. Shupihui, 16: 451-463.

NAGAR, R., 1997 - Communal Places and the Politics of Multiple Identities: The Case of Tanzanian Asians. Ecumene, 4 (1): 3-26. 
NEWING, H. \& WAHL, L., 2004 - Benefiting Local Populations? Communal Reserves in Peru. Cultural Survival Quarterly, 28 (1): 38-41.

OFFEN, K. H., 2003a - Narrating Place and Identity, or Mapping Miskitu Land Claims in Northeastern Nicaragua. Human Organization, 62 (4): 382-392.

OFFEN, K. H., 2003b - The Territorial Turn: Making Black Territories in Pacific Colombia. Journal of Latin American Geography, 2 (1): 43-73.

PACURI, F. \& MOORE, T., 1992 - Los conflictos entre mineros auríferos y el pueblo arakmbut en Madre de Dios, Perú; Puerto Maldonado: Centro Eori de Investigación y Promoción Regional. Documento inédito.

PINEDO, D., 2014 - The Politics of Sociality: Social Networks and Indigenous Mobilization in Peruvian Amazonia; Gainesville: University of Florida. Tesis doctoral.

PINEDO, D., 2017 - The Making of the Amazonian Subject: State Formation and Indigenous Mobilization in Lowland Peru. Latin American and Caribbean Ethnic Studies, 12 (1): 2-24.

QUIJANO, A., 2000 - Coloniality of Power, Eurocentrism, and Latin America. Nepantla: Views from South, 1 (3): 533-580.

SACK, R. D., 1986 - Human Territoriality: Its Theory and History, 256 pp.; Cambridge: Cambridge University Press.

SLETTO, B. I., 2009 - 'We Drew What We Imagined': Participatory Mapping, Performance, and the Arts of Landscape Making. Current Anthropology, 50 (4): 443-476.

SMITH, R. C. \& PINEDO, D., 2003 - Comunidades y áreas naturales protegidas en la Amazonía peruana. Debate Agrario, 36: 15-37.

TROUILLOT, M., 2001 - The Anthropology of the State in the Age of Globalization: Close Encounters of the Deceptive Kind. Current Anthropology, 42 (1): 125-138.

VANDERGEEST, P. \& PELUSO, N. L., 1995 - Territorialization and State Power in Thailand. Theory and Society, 24 (3): 385-426.

VARESE, S., 2018 - De la represión a la revolución: el velasquismo en la Amazonía (19681975). In: La revolución peculiar: repensando el gobierno militar de Velasco (C. Aguirre \& P. Drinot, eds.): 419-441; Lima: Instituto de Estudios Peruanos.

WAINWRIGHT, J. \& BRYAN, J., 2009 - Geography, Territory, Property: Postcolonial Reflections on Indigenous Counter-mapping in Nicaragua and Belize. Cultural Geographies, 16 (2): 153-178. 